\title{
Candidal Carriage in Saliva and Subgingival plaque among smokers and non-smokers with Chronic Periodontitis - A Cross-Sectional Study
}

\author{
Gayathri Krishnan ${ }^{1}$, Dilip Naik ${ }^{1}$, Ashita Uppoor ${ }^{\text {Corresp., }}{ }^{1}$, Sangeeta U Nayak ${ }^{1}$, Srikala Baliga ${ }^{2}$, Abhiram Maddi ${ }^{3}$ \\ ${ }^{1}$ Department of Periodontology, Manipal College of Dental Sciences (MCODS) Mangalore, Manipal Academy of Higher Education, Mangalore, Karnataka, \\ India \\ 2 Department of Microbiology, Kasturba Medical College (KMC) Mangalore, Manipal Academy of Higher Education, Mangalore, Karnataka, India \\ 3 Periodontics \& Endodontics, School of Dental Medicine, State University of New York at Buffalo, Buffalo, New York, United States \\ Corresponding Author: Ashita Uppoor \\ Email address: ashita.uppoor@manipal.edu
}

Back ground and Objectives: Studies of gum or periodontal disease have focused mainly on bacterial pathogens. However, information related to fungal species in the saliva and subgingival mileu is particularly lacking in smokers with periodontitis. This crosssectional study compared the prevalence of various Candida species in saliva and subgingival plaque samples of smokers and non-smokers with periodontal disease. Methodology: Study subjects were recruited into 3 Groups - Group 1: Smokers with chronic periodontitis $(\mathrm{N}=30)$, Group 2: Non-smokers with chronic periodontitis $(\mathrm{N}=30)$ and Group 3: Healthy controls $(\mathrm{N}=30)$. Clinical parameters recorded included plaque index $(\mathrm{PI})$, gingival index $(\mathrm{GI})$, periodontal probing depth (PPD) and clinical attachment loss (CAL). Saliva and subgingival plaque samples were collected from subjects from the above groups. The collected samples were processed for isolation and identification of various Candida species using CHROMagar chromogenic media. Additionally, antifungal susceptibility tests were performed for the isolated Candida species in order to assess antifungal drug resistance to fluconazole and voriconazole. Results: Prevalence of Candida species in saliva samples was quantified as 76.6\% in Group 1, 73.3\% in Group 2 and $36.6 \%$ in Group 3 and statistically significant differences were observed between groups $1 \& 3$. Prevalence of Candida species in subgingival plaque samples was quantified as $73.3 \%$ in Group 1, 66.6\% in Group 2 and $60 \%$ in Group 3 and no statistically significant differences were observed between groups. Candida albicans was the most frequently isolated species followed by Candida krusei and Candida tropicalis. A positive correlation was observed for smoking exposure, pack years and Candida colonization. A marginally significant positive correlation was observed between Candida colonization and increasing pocket depth and attachment loss. Antifungal drug resistance was mainly observed for 
Candida krusei in both saliva and subgingival plaque samples. Conclusion: Based on the results we can conclude that oral candidal carriage is significantly increased in smokers with periodontal disease. Mechanistic studies are needed to understand the importance of Candida species in periodontal disease. 
1 Candidal Carriage in Saliva and Subgingival Plaque among Smokers and Non-smokers

2 with Chronic Periodontitis - A Cross-Sectional Study

3 Gayathri Krishnan ${ }^{1}$, Dilip Naik ${ }^{1}$, Ashita Uppoor ${ }^{1 *}$, Sangeeta U Nayak ${ }^{1}$, Srikala Baliga ${ }^{2}$, Abhiram

4 Maddi $^{3}$

$5{ }^{1}$ Department of Periodontology, Manipal College of Dental Sciences (MCODS) Mangalore,

6 Manipal Academy of Higher Education, Mangalore, Karnataka, India

7 2Department of Microbiology, Kasturba Medical College, Mangalore, Manipal Academy of

8 Higher Education, Mangalore, Karnataka, India

$9{ }^{3}$ Periodontics \& Endodontics, School of Dental Medicine, State University of New York at

10 Buffalo, Buffalo, NY.

11 Running Title: Assessment of Candida in smokers with chronic periodontitis

12 Key Words: Chronic periodontitis, Periodontal disease, Smoking, Saliva, Subgingival plaque,

13 Candida species, Candida albicans, Candida krucei, antifungal susceptibility

14 *Correspondence:

15 Dr. Ashita S Uppoor BDS, MDS,

16 Professor and Associate Dean

17 Department of Periodontology

18 Manipal College of Dental Sciences (MCODS) Mangalore,

19 Manipal Academy of Higher Education, Mangalore, Karnataka, India.

20 E-mail: ashita.uppoor@manipal.edu

21 Ph: +919880038082 
Fax: +918242422653

\section{ABSTRACT}

24 Back ground and Objectives: Studies of gum or periodontal disease have focused mainly on

25 bacterial pathogens. However, information related to fungal species in the saliva and subgingival

26 mileu is particularly lacking in smokers with periodontitis. This cross-sectional study compared

27 the prevalence of various Candida species in saliva and subgingival plaque samples of smokers

28 and non-smokers with periodontal disease. Methodology: Study subjects were recruited into 3

29 Groups - Group 1: Smokers with chronic periodontitis (N=30), Group 2: Non-smokers with

30 chronic periodontitis $(\mathrm{N}=30)$ and Group 3: Healthy controls $(\mathrm{N}=30)$. Clinical parameters recorded

31 included plaque index (PI), gingival index (GI), periodontal probing depth (PPD) and clinical

32 attachment loss (CAL). Saliva and subgingival plaque samples were collected from subjects from

33 the above groups. The collected samples were processed for isolation and identification of various

34 Candida species using CHROMagar chromogenic media. Additionally, antifungal susceptibility

35 tests were performed for the isolated Candida species in order to assess antifungal drug resistance

36 to fluconazole and voriconazole.

37 Results: Prevalence of Candida species in saliva samples was quantified as $76.6 \%$ in Group 1, $3873.3 \%$ in Group 2 and $36.6 \%$ in Group 3 and statistically significant differences were observed

39 between groups $1 \& 3$. Prevalence of Candida species in subgingival plaque samples was

40 quantified as $73.3 \%$ in Group 1, 66.6\% in Group 2 and $60 \%$ in Group 3 and no statistically

41 significant differences were observed between groups. Candida albicans was the most frequently

42 isolated species followed by Candida krusei and Candida tropicalis. A positive correlation was

43 observed for smoking exposure, pack years and Candida colonization. A marginally significant

44 positive correlation between Candida colonization and increasing pocket depth and attachment 
45 loss was noted. Antifungal drug resistance was mainly observed for Candida krusei in both saliva

46 and subgingival plaque samples.

47 Conclusion: Based on the results we can conclude that oral candidal carriage is significantly

48 increased in smokers with periodontal disease. Mechanistic studies are needed to understand the

49 importance of Candida species in periodontal disease.

\section{1. INTRODUCTION}

51 Periodontal disease is a multifactorial disease and associated with complex microbial interactions.

52 Periodontopathogenic bacteria, mainly red-complex bacteria (Porphyromonas gingivalis,

53 Treponema denticola, and Tannerella forsythia) and Aggregatibacter actinomycetemcomitans

54 have been implicated as the etiological agents for periodontal disease. ${ }^{1}$ However, recent studies

55 that performed microbiome sequencing indicate that more than 700 species of bacteria are part of

56 the oral milieu. ${ }^{2-5}$ Some of these species are beneficial and contribute to a healthy oral homeostasis,

57 while others are associated with disease. Keystone species are considered as pathogenic

58 components of the disease-inducing microbiota. ${ }^{6}$ Proinflammatory characteristics and interspecies

59 signaling result in community shifts in microbiota along with a host response leading to

60 periodontal tissue destruction. ${ }^{7,8}$ However, a third of the oral microbial species-level taxa remain

61 uncultured, including many of the disease-associated microbial organisms. ${ }^{8}$ This indicates that in

62 addition to red complex bacteria other species may play a role pathogenesis of periodontitis.

There has been a rising interest in understanding the role of fungi in dental plaque and

64 periodontal disease. Recent microbiome analyses have indicated that Candida albicans is the most

65 abundantly found opportunistic fungus in the saliva as well as subgingival plaque. ${ }^{9,10}$ Many

66 predisposing factors (local and general) cause an increase in Candida colonization causing damage 
67 to the oral mucosa. They include cigarette smoking, poor oral hygiene, hypovitaminosis, dentures,

68 pregnancy, HIV infection, diabetes mellitus and debilitated patients on antibiotics, steroids or

69 cytotoxic therapy. ${ }^{11}$ In a healthy state, Candida species reside on the buccal mucosa, tongue, palate,

70 and saliva. They have been recovered in $40-60 \%$ of healthy oral carriers. Although candida

71 colonization is rarely found in subgingival sites in healthy subjects, Candida species have often

72 been isolated from periodontal pockets. ${ }^{12}$ Few earlier studies explored the occurrence and probable

73 role of Candida species in periodontal diseases. ${ }^{12-14}$ These studies used culture methods and/or

74 genotypic methods to isolate and identify Candida species in the oral and plaque samples. They

75 concluded the increased presence of $C$. albicans in periodontal pockets as compared to healthy

76 sites, although there was no statistical significance. However, C. albicans was found to be

77 associated with severity of periodontal disease. ${ }^{12-14}$

Smoking is a major environmental factor that significantly increases the risk of periodontal

disease. ${ }^{15,16}$ Smoking is known to affect periodontal attachment loss via several pathways including microcirculatory, inflammatory and immune-mediated mechanisms. However, the exact mechanisms of the effect of smoking at the cellular and molecular level are not clear. ${ }^{17}$ Smoking

82 has long been considered to affect the microbial milieu by favoring periodontal pathogenic bacteria. However, recent studies have shown that there is no significant difference in the

84 supragingival or subgingival microbial milieu between smokers and non-smokers. ${ }^{17,18}$ Most of 85 these studies have focused on bacterial species and the data on fungal species is particularly 86 lacking. Besides the relationship between smoking, periodontitis and oral Candida colonization is 87 unclear. Moreover, microbial profiling in aggressive periodontitis showed an increased prevalence 88 of Candida in patients who were smokers. ${ }^{19}$ Hence this study aimed to assess the quantitative and 
89 qualitative oral colonization of Candida species in saliva and subgingival sites among smokers \&

90 non-smokers with chronic periodontitis.

\section{2. MATERIALS \& METHODS:}

\section{$92 \quad 2.1$ Study Participants}

93 The present cross-sectional study was performed in the Department of Periodontology, Manipal

94 College of Dental Sciences, Mangalore Manipal Academy of Higher Education, Manipal,

95 Karnataka, India. A total of 90 male patients in the age range of $20-50$ years (mean age $35.88 \pm 8.39$

96 yrs) reporting to the outpatient section were recruited based on the following criteria between Jan

972015 to Jun 2016. This was due to the extremely low incidence of smoking in females as seen in

98 the Indian population Inclusion criteria for this study were; patients with moderate to severe

99 generalized chronic periodontitis (i.e. $>5 \mathrm{~mm}$ loss of attachment, $>4 \mathrm{~mm}$ probing depth in at least

$10030 \%$ of the sites, bleeding upon probing present in mouth) (American Academy of Periodontics,

101 1999). ${ }^{20}$ Smokers included those who have smoked 100 cigarettes in a lifetime while those who

102 never smoked were included in the non-smoker Groups. ${ }^{8}$ The exclusion criteria were; subjects

103 who were on medication such as corticosteroids, antibiotics, or medication for xerostomia,

104 antifungal agents / antiseptic mouthwash over past six months; subjects who reported any systemic

105 predisposing factor for oral candidiasis such as diabetes mellitus or anemia, those wearing

106 removable dental prosthesis or orthodontic appliance, subjects using smokeless tobacco and

107 subjects having aggressive periodontitis, necrotizing ulcerative gingivitis or necrotizing ulcerative

108 periodontitis. Approval was obtained from the Institutional Ethics Committee and Review board,

109 (Protocol no: 14129). All the participants signed the informed consent preceding to

110 commencement of the study following the Helsinki agreement.

\section{Patient Groups:}


112 After the sample size calculation, a non-probability convenience sampling was done, the study

113 participants were divided into 3 Groups of 30 participants each: Group 1: Smokers with

114 periodontitis, Group 2: Non-smokers with periodontitis, Group 3: Healthy patients (non-

115 smokers) with no periodontal disease (controls).

116 Smoking Status \& Nicotine Dependence:

117 Smoking history was elicited from the patient based on a questionnaire. Smoking exposure was

118 calculated as the number of cigarettes per day $\times$ duration (in years). Pack years were calculated

119 according to the formula: (No. of cigarettes smoked/day) * (No. of years smoked) / (No. of

120 cigarettes in one pack). Fagerstrom test was performed to assess the intensity of physical

121 dependence on nicotine. ${ }^{21}$ Nicotine dependence of subjects was assessed based on smoking habits

122 and frequency. ${ }^{21}$

123 2.2 Clinical Examination: A complete clinical examination of full mouth was done using a

124 periodontal probe (Williams's markings, Hu-Freidy, Chicago, IL, USA\& recorded by two

125 experienced \& calibrated examiners for each patient. The following clinical parameters were

126 recorded: Plaque index (PI) ${ }^{22}$, Gingival index (GI) ${ }^{23}$, Probing pocket depth (PPD), Clinical

127 attachment loss (CAL), Bleeding upon probing(BOP)-Modified sulcus bleeding index ${ }^{24}$ : Probing

128 pocket depth and level of clinical attachment at six sites around each tooth (mesio-buccal, buccal,

129 disto-buccal, mesio-lingual, lingual, and disto-lingual) were assessed to the nearest millimeter

130 using Williams's periodontal probe. Clinical attachment loss was measured in millimeters from

131 Cemento Enamel Junction (CEJ) to the base of the periodontal pocket. All measurements were

132 taken by a single examiner.

\section{2.3 Microbiological Sampling and Analysis:}

\section{Sample Collection}


135 The sample collection was done by the same clinical examiner between 9 AM to 11 AM. Subjects

136 were refrained from food intake, drink, any oral hygiene procedure or smoking for at least 1 hour

137 before sample collection. Saliva collection was done as previously described using concentrated

138 oral rinse (COR) technique. ${ }^{25}$ Briefly, each study participant was provided with $10 \mathrm{ml}$ of sterile

139 phosphate-buffered saline (PBS 0.1M, pH 7.2) and asked to rinse their mouth thoroughly for 60

140 seconds and then to expectorate in a sterile plastic tube. The collected samples were processed

141 immediately in the Microbiology department of the institute. Subgingival plaque was collected as

142 previously described. ${ }^{14}$ For each patient, after superficial cleaning of the sites with cotton pellets

143 and removal of supragingival plaque the subgingival plaque samples were collected from the three

144 sites with the deepest periodontal pockets by using Gracey curettes and pooled into one container.

145 The collected subgingival plaque samples were transferred to PBS in sterile capped containers and

146 transported on ice to the Microbiology department for microbiological analysis of Candida

147 species.

148 Assessment of Candida Prevalence \& Relative Abundance:

149 Quantification of candida carriage in the samples was performed as described previously. ${ }^{26}$

150 Briefly, samples were centrifuged for 10 minutes at $1000 \mathrm{rpm}$ and the supernatant discarded. The

151 pellet was re-suspended in $1 \mathrm{ml}$ of PBS. A sample volume of 20 microlitre was taken and streaked

152 using a sterile glass spreader onto Sabourauds Dextrose Agar plates in duplicates for culture and

153 incubated at $37^{\circ} \mathrm{C}$. After 48 hours, the Candida colonies were counted and the colony-forming unit

154 per $\mathrm{ml}(\mathrm{CFU} / \mathrm{ml})$ of saliva and subgingival plaque was calculated. Candida sub speciation was

155 determined using CHROMagar cultures. The Candida isolates from the culture plates were

156 inoculated onto CHROMagar plates and incubated at $37^{\circ} \mathrm{C}$ for 48 hours. Different Candida species

157 imparted different colors to colonies when incubated in CHROMagar chromogenic medium: $C$. 
158 albicans - light green colonies with pale edges; C. parapsilosis - pale cream colonies; C. krusei-

159 spreading rose pink colonies with broad, pale edges; $C$. tropicalis - bluish-green colonies; $C$.

160 glabrata - dark pink colonies with pale edges. After determination of Candida subspecies,

161 antifungal susceptibility testing was done for the isolated species.

\section{$162 \quad 2.4$ Antifungal Disc Diffusion Susceptibility Testing}

163 Each sample isolate was subcultured on Sabouraud dextrose agar and incubated at $35^{\circ} \mathrm{C}$ twice

164 before testing to ensure clarity and optimum growth. For antifungal testing the Clinical and

165 Laboratory Standards Institute (CLSI) reference method for broth dilution antifungal susceptibility

166 testing of yeasts was used. ${ }^{27}$ Briefly, Mueller-Hinton agar enhanced with $2 \%$ glucose and $0.5 \mathrm{~g}$ of

167 Methylene Blue (MB) per ml were used and discs were applied to determine susceptibility. In

168 distilled water, standard solutions of glucose $(0.4 \mathrm{~g} / \mathrm{ml})$ and $\mathrm{MB}(5 \mathrm{mg} / \mathrm{ml})$ were added and the

169 medium was prepared. They were sterilized and stored at $4^{\circ} \mathrm{C}$. The Mueller Hilton agar plates were

170 made using the Glucose Methylene Blue (GMB) standard solution $(2.9 \mathrm{ml})$ and allowed to absorb

171 for 4 to $6 \mathrm{~h}$ before inoculation. The inoculum was prepared using 4-5 representative colonies of

172 the Candida sp., and suspending in $5 \mathrm{ml}$ of sterile normal saline to match $0.5 \mathrm{McFarland}$ standard.

173 Candida albicans ATCC 90028 strain was utilized as reference strain for disk diffusion testing. A

174 sterile cotton swab dipped into inoculum was inoculated onto agar plates by evenly streaking in

175 all directions over the whole surface area of the agar plates. The plates were allowed to dry for 15

$176 \mathrm{~min}$ and the disks with fluconazole and voriconazole are placed onto each inoculated plate, and

177 the plates were incubated at $35^{\circ} \mathrm{C}$. Then readings were taken after 24 and $48 \mathrm{~h}$. Inhibitory zone

178 (zone of inhibition) diameters for the disks were measured and interpreted as follows: Susceptible

179 - Zone diameters of $19 \mathrm{~mm}$ seen, Susceptible-dose dependent - Zone diameters of 15 to $18 \mathrm{~mm}$

180 seen, Resistance - Zone diameters of $14 \mathrm{~mm}$ seen. ${ }^{28}$ The readings were taken after 24 and $48 \mathrm{hrs}$. 
181 The diameter of zone of inhibition was measured at the transitional point where growth abruptly

182 decreases and was determined by reduction in colony size, number and density.

183 2.5 Statistical analysis:

184 Data within each Group were expressed as mean \pm standard deviation (SD) in 90 patients. All

185 calculations were performed using SPSS software v 20.0 (SPSS Inc., Chicago, IL, USA).

186 Comparison of the clinical parameters and microbiological parameters between the control and

187 two experimental Groups were performed by one way ANOVA Post hoc power analysis was done

188 by applying the Tukey test. A $p$-value $<0.05$ was considered as statistically significant. Association

189 of smoking as a confounding factor with Candida colonization and periodontitis was performed

190 using Pearson's correlation test.

191

192 RESULTS:

\section{3.1. Clinical parameters of periodontal disease}

194 Since the study participants were age-matched, the comparison of age distribution within the

195 Groups showed no statistically significant difference. The mean plaque index (PI) score of Groups

1961,2 and 3 was $1.62 \pm 0.48,1.51 \pm 0.51$ and $0.67 \pm 0.22$ respectively. No significant differences

197 were seen for PI. This suggests that the effect of tobacco smoking on the periodontium were

198 independent of the plaque level. The gingival index (GI) for Groups 1, 2 and 3 was $1.53 \pm 0.29$,

$1991.64 \pm 0.43$ and $0.79 \pm 0.18$ respectively. There was a statistically significant difference for GI

200 between Groups 1 and 2. The bleeding on probing (BOP) score for Groups 1, 2 and 3 was 1.6, 1.9

201 and 1.03 respectively and was found to be significantly different between all Groups. The probing

202 pocket depth (PPD) among Groups 1 and 2 was $6.64 \pm 0.60 \mathrm{~mm}$ and $6.85 \pm 0.85 \mathrm{~mm}$ respectively.

203 The comparison of PPD within the Groups showed no significant difference between Groups 1

Peer) reviewing PDF | (2019:07:39057:2:0:NEW 7 Dec 2019) 
204 and 2. The clinical attachment loss (CAL) among Groups 1 and 2 was $7.06 \pm 0.71 \mathrm{~mm}$ and $7.18 \pm$

$2051.17 \mathrm{~mm}$ respectively. The comparisons of CAL showed no statistically significant difference

206 between Groups 1 and 2.

207 3.2 Relative abundance of Candida species in saliva and subgingival plaque samples

208 Candida species were isolated from $76.6 \%$ of patients in Group 1, 73.3\% in Group 2 and $36.6 \%$

209 of patients in Group 3 from saliva samples. The mean $\mathrm{CFU} / \mathrm{ml}$ was $13 \pm 13.7,10 \pm 12.2$ and $3.7 \pm$

2103.1 in Groups 1, 2 and 3 respectively. When compared among the Groups, Candida colonization

211 showed statistically significant differences among Groups 1 and $3(\mathrm{p}<0.004)$. This data indicates

212 that in saliva, Candida species are significantly higher in smokers with periodontitis in comparison

213 to healthy controls. Candida species were isolated in 73.3\% subjects in Group 1, 66.6\% in Group

2142 and $60 \%$ in Group 3 from subgingival plaque. The mean CFU/ml was $8.1 \pm 7.383,7.47 \pm 8.83$

215 and $4.43 \pm 4.88$ and in Groups 1, 2 and 3 respectively. However, statistical analyses did not indicate

216 any significant differences in subgingival plaque samples between Groups. A total of 5 different

217 Candida subspecies were isolated among the 3 study Groups from saliva and included C. albicans,

218 C. tropicalis, C. krusei, C. glabrata and C. parapsilosis. However, only 4 different subspecies

219 were isolated from subgingival plaque and included C. albicans, C. krusei, C. tropicalis and C.

220 glabrata. In saliva, C. albicans was the most commonly isolated subspecies among all Groups,

221 while C. krusei and C. tropicalis were next followed by C. glabrata which was the least frequently

222 isolated species. Interestingly, C. parapsilosis was isolated from saliva of only one subject in the

223 smokers with periodontitis Group (Group 1). Even in subgingival plaque, C. albicans was the most

224 commonly isolated subspecies among all Groups. C. krusei and C. tropicalis were the second and

225 third most frequently isolated subspecies for Groups 1,2 and 3 respectively from subgingival

226 plaque. Among Groups 1 and 2, C. glabrata was isolated from 3.30\% subjects. (Table 1 and Figure 
227 1). The antifungal susceptibility testing showed that the majority of the isolated Candida

228 subspecies were susceptible to the drugs, fluconazole and voricanozole. Interestingly, C. krusei

229 isolates showed antifungal resistance in both saliva and subgingival plaque samples. (Table 2)

\section{$230 \quad 3.3$ Correlation among clinical parameters and microbiological analysis of samples}

231 Correlation tests were performed to examine the correlation between smoking exposure, nicotine

232 dependence, pack year, PPD and CAL. There was fair correlation between smoking exposure, (p 233 0.311) nicotine dependence, ( $\mathrm{p}$ 0.291), pack years ( $\mathrm{p} 0.150$ ) and Candida colonization in saliva

234 and subgingival sites. This data indicates that duration of smoking and number of cigarettes

235 smoked correlated positively with Candida colonization. There was also positive marginally

236 significant correlation between Candida colonization and increased PPD and CAL. Deeper pocket 237 depths correlated with increased prevalence of complex Candida species (Table 3, Figures $2 \& 3$ ).

239 4. DISCUSSION:

240 In the current study, an association between the subgingival colonization of Candida species,

241 especially C. albicans, in smokers with periodontitis was noted. In addition, a great diversity of

242 Candida species, such as Candida tropicalis, Candida krusei, Candida parapsilosis, Candida

243 glabrata were also associated with Candida albicans as observed in previous studies. ${ }^{13}$ This

244 further confirms that advanced forms of periodontitis and tobacco smoking are associated with 245 complex yeast communities in deep periodontal pockets. Also, a great diversity of Candida

246 species, such as C. tropicalis, C. krusei, C. parapsilosis, C. glabrata were found to be associated

247 with C. albicans. Past studies that assessed the microbial profile of smokers in aggressive 248 periodontitis patients found increased levels of C. albicans in periodontal pockets. ${ }^{19}, 28$

249 Furthermore, tobacco users were found to have elevated levels of C. albicans in saliva. ${ }^{30}$ 
In the present study, smoking was found to be independent of plaque levels in agreement

251

252

253

254

255 256

with various other studies. ${ }^{31,32}$ Clinical signs of gingival inflammation such as bleeding, redness, and exudation were not evident in smokers with periodontitis (Group 1). This could be due to peripheral vasoconstriction of blood vessels caused by smoking. The localized vasoconstrictive actions of nicotine may also be responsible for the reduced vascular supply in gingiva. Increased

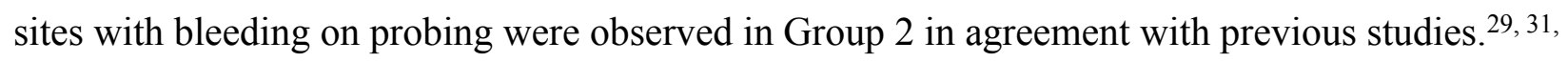
32

Saliva harbors large concentration of Candida species in the disease process and provides a niche and reflects the changes in nature and behavior of the underlying disease process. ${ }^{33}$ Saliva can be a good means of identifying oral candidal carriage. Besides, it has been speculated that the percentage of Candida species in the subgingival plaque is proportional to some bacterial periodontopathogens, suggesting the probable role for Candida species in the pathogenesis of periodontal disease. ${ }^{34}$ In the present study, the overall candidal carriage was significantly higher in the saliva of smokers with periodontal disease concurrent to previous studies. ${ }^{11,26,32}$ This may be due to low salivary flow rate and increased dryness of the mouth due to long term smoking altered host response and vasculature is seen among smokers. This, in turn, may favor the increase in Candida colonization. In subgingival plaque samples, Candida colonization was seen among all the groups concurrent to earlier studies. ${ }^{13,14}$ However, comparison between the groups was not statistically significant. This could be attributed to the relatively lower amounts of subgingival plaque samples collected as compared to the saliva samples and also the individual variations among different study subjects. Candida density was also very heterogeneous in the saliva and subgingival plaque samples. These differences could be attributed to the altered immunological 
272 status due to smoking and chronic systemic inflammation from periodontitis in these subjects

273 similar to earlier studies. ${ }^{35,36 .}$

In the present study, C. albicans was the most commonly detected Candida species in

275 saliva and plaque samples, which is similar to data from previous studies. ${ }^{36-39}$ Decreased

276 colonization of $C$. albicans was seen in subgingival plaque compared to saliva. This could be due

277 to site-specificity and minimal amount of species which go undetected. C. glabrata, C. tropicalis,

278 C. krusei, and C. parapsilosis were the other candida subspecies found in the present study. $C$.

279 krusei and C. tropicalis were higher in Group1 \& 2 (Table 1) similar to earlier studies. ${ }^{40,41}$ This

280 suggests that this species may be particularly adapted to oral colonization as a constituent of

281 normal human oral flora, with a potential to cause clinical infection. Studies have shown that $C$.

282 krusei and C. tropicalis are more virulent, possibly due to their capacity to adhere to epithelial

283 cells in vitro and secretion of proteinase. ${ }^{42}$ It was also noteworthy that specific Candida subspecies

284 obtained in saliva sample were not seen in subgingival plaque samples of the same subject. This

285 shows that individual sites are colonized by distinct Candida species (site-specific) which are

286 otherwise not seen on the oral mucosa or periodontal pockets, or in healthy patients as observed

287 previously. ${ }^{14}$

The Fagerstrom test revealed high nicotine dependence (score of $8-10$ ) in 10 patients and moderate dependence score (5-8) in 6 patients and also that Candida colonization increased with nicotine dependence. An increase in smoking duration and number of cigarettes showed an

291 increase in Candida colonization in saliva and subgingival plaque (Table 3). Cigarette smoking

292 possibly increases keratinization of epithelium and enhancement of hydrophobicity and is 293 recognized as a predisposing factor for oral yeast carriage. ${ }^{43}$ There was a marginal positive 
294 correlation in candida colonization with increasing pocket depth and attachment loss. This may be

295 due to a small sample size but is concurrent with other studies. ${ }^{13,14,44}$

C. albicans has a predominant role in the immune evasion and adhesion to the periodontal

297 tissues. The periodontal pockets provide a suitable environment for change in microflora and 298 predispose periodontal destruction. C. albicans may exacerbate periodontitis by enhancing the 299 invasion of host cells by anaerobic bacteria such as $P$. gingivalis. $P$ gingivalis, can alter the local 300 immune response and hypothetically may influence opportunistic organisms like C. albicans 301 inhabiting the same subgingival niche and thus there could be a symbiotic and synergistic 302 relationship between the two organisms. Moreover, C. albicans can produce proteinases that 303 destroy major extracellular matrices and basement membrane components. ${ }^{42}$ The current study 304 indicates that smoking and periodontitis affects oral colonization of Candida species. Smoking 305 impairs host responses to periodontal therapy. Additionally, a recent study in a rat model revealed 306 that long term smoking attenuates host defense against $C$. albicans by suppressing NLRP3 307 inflammosome. ${ }^{45}$ Periodontal pathogens combined with some Candida species are resistant to 308 short-term periodontal therapy. ${ }^{43}$ Furthermore, C. albicans has been shown to increase antibiotic 309 tolerance of oral plaque bacteria like Streptococcus gordonii. ${ }^{46}$ Additionally, it was also found 310 that binding of $S$. gordonii to the cell wall of C. albicans was important for biofilm formation and 311 antibiotic tolerance of S. gordonii. ${ }^{46}$ Antifungal susceptibility testing, indicated that C. krusei was

312 found to be resistant to fluconazole, in both subgingival plaque and saliva concurrent to previous 313 studies. ${ }^{47,48}$ But the other Candida subspecies isolated in this study were not susceptible to either

314 fluconazole or voricanozole. It needs to be examined if this antifungal susceptibility of oral

315 Candida species is variable depending on genetic and geographical factors. Such information may

316 be useful in predicting the occurrence of systemic candidiasis. 
A major limitation of this study is related to the gender of the subjects. Only male subjects

318 were included here as the number of women smokers is low in India with a prevalence of $4 \% .{ }^{49}$

319 Also, since it is cross sectional study, the cause and effect relationship cannot be established. Given

320 the findings of the current investigation, further large multicenter studies are required to examine

321 the effect of smoking on oral candidal carriage in standardized study populations with long term

322 follow up and interventional treatment. Also, future studies should incorporate variables such as

323 salivary flow, saliva composition, and Candida adhesion to oral epithelial cells for a more

324 comprehensive analysis.

325

\section{5. CONCLUSION}

327 From the analysis of the results and within limitations of the present study, it can be concluded

328 that oral candidal burden is increased in smokers with chronic periodontitis as compared to

329 smokers without periodontal disease. It needs to be investigated further if this increase in candidal

330 burden in smokers with chronic periodontitis has any relationship with etiopathogenesis of

331 periodontal disease in this population.

332 Acknowledgments: None

\section{REFERENCES}

334 1. Socransky SS, Haffajee AD. Periodontal microbial ecology. J. Periodontol 2000;

$335 \quad 2005 ; 38: 135-187$.

336 2. Griffen AL, Beall CJ, Campbell JH, Firestone ND, Kumar PS, Yang ZK, Podar M, Leys 
16S pyro sequencing. ISME J. 2012 Jun;6(6):1176-85. doi: 10.1038/ismej.2011.191. community biomass and inflammation. ISME J. 2013 May;7(5):1016-25. doi: 10.1038/ismej.2012.174. Epub 2013 Jan 10. PMID: 23303375; PMCID: PMC3635234. Levels of Candidate Periodontal Pathogens in Subgingival Biofilm. J Dent Res. 2016 Jun;95(6):711-8. doi: 10.1177/0022034516634619. Epub 2016 Mar 2. PMID: 26936213; PMCID: PMC4924544.

5. Liu B, Faller LL, Klitgord N, Mazumdar V, Ghodsi M, Sommer DD, Gibbons TR, Treangen TJ, Chang YC, Li S, Stine OC, Hasturk H, Kasif S, Segrè D, Pop M, Amar S. One. 2012;7(6):e37919. doi: 10.1371/journal.pone.0037919. Epub 2012 Jun 4. PMID: $22675498 ;$ PMCID: PMC3366996.

6. Stone VN, Xu P. Targeted antimicrobial therapy in the microbiome era. Mol Oral Microbiol. 2017;32:446-454.

7. Lopez R, Hujoel P, Belibasakis GN. On putative periodontal pathogens: a epidemiological perspective. Virulence. 2015;6:249-257. between blacks and whites. Drug Alcohol Depend. 1993;32:119-125.

9. Ghannoum MA, Jurevic RJ, Mukherjee PK, Cui F, Sikaroodi M, Naqvi A, Gillevet PM. Characterization of the oral fungal microbiome (mycobiome) in healthy individuals. 
PLoS Pathog. 2010 Jan 8;6(1):e1000713. doi: 10.1371/journal.ppat.1000713. PMID: 20072605; PMCID: PMC2795202.

363 10. Vesty A, Biswas K, Taylor MW, Gear K, Douglas RG. Evaluating the Impact of DNA

364 Extraction Method on the Representation of Human Oral Bacterial and Fungal

$365 \quad$ Communities. PLoS One. 2017;12:e0169877.

366 11. Muzurović S, Hukić M, Babajić E, Smajić R. The relationship between cigarette smoking 367 and oral colonization with Candida species in healthy adult subjects. Med Glas (Zenica). $368 \quad 2013 ; 10: 397-399$.

369 12. Samaranayake L. Commensal oral Candida in Asian cohorts. Int. J. Oral Sci. 2009;1:2-5.

370 13. Canabarro A, Valle C, Farias MR, Santos FB, Lazera M, Wanke B. Association of 371 subgingival colonization of Candida albicans and other yeasts with severity of chronic periodontitis. J Periodontal Res. 2013;48:428-432.

14. McManus BA, Maguire R, Cashin PJ, Claffey N, Flint S, Abdulrahim MH, Coleman DC. Enrichment of multilocus sequence typing clade 1 with oral Candida albicans isolates in patients with untreated periodontitis. J Clin Microbiol. 2012 Oct;50(10):3335-44. doi: 10.1128/JCM.01532-12. Epub 2012 Aug 8. PMID: 22875886; PMCID: PMC3457439.

15. Tomar SL, Asma S. Smoking-attributable periodontitis in the United States; findings

16. Johnson GK, Hill M. Cigarette smoking and the periodontal patient. J Periodontol. 2004; 75:196-209.

17. Nociti FH Jr, Casati MZ, Duarte PM. Current perspective of the impact of smoking on the progression and treatment of periodontitis. Periodontol 2000. 2015;67:187-210. 
384 18. Kibayashi M, Tanaka M, Nishida N, Kuboniwa M, Kataoka K, Nagata H, Nakayama K,

385 Morimoto K, Shizukuishi S. Longitudinal study of the association between smoking

386 as a periodontitis risk and salivary biomarkers related to periodontitis. J Periodontol.

387 2007 May; 78(5):859-67. PubMed PMID: 17470019.

388 19. Kamma JJ, Nakou M, Baehni PC. Clinical and microbiological characteristics of smokers

389 with early-onset periodontitis. J Periodontal Res. 1999;34:25-33.

390 20. Armitage GC. Periodontal diagnoses and classification of periodontal diseases.

$391 \quad$ Periodontology 2000. 2004;34:9-21.

392 21. Heatherton TF, Kozlowski LT, Frecker RC, Fagerstrom KO. The Fagerstrom Test for

393 Nicotine Dependence: A revision of the Fagerstrom Tolerance Questionnaire. $\mathrm{Br} J$

394 Addict. 1991;86:1119-1127.

395 22. Silness J, Loe H. Periodontal disease in pregnancy. II. Correlation between oral hygiene 396 and periodontal condition. Acta Odontol Scand 1964;22:121-135.

397 23. Loe H, Silness J. Periodontal disease in pregnancy. I. Prevalence and severity. Acta $398 \quad$ Odontol Scand 1963;21:533-551.

399 24. Mombelli A, van Oosten MA, Schurch E Jr, Land NP. The microbiota associated

400 with successful or failing osseointegrated titanium implants. Oral Microbiol

401 Immunol. 1987 Dec;2(4):145-51. PubMed PMID: 3507627.

402 25. Nikawa H, Hayashi S, Nikawa Y, Hamada T, Samaranayake LP. Interactions between

403 denture lining material, protein pellicles, and Candida albicans. Arch Oral Biol.

$404 \quad 1993 ; 38: 631-634$.

405 26. Darwazeh AM, Al-Dwairi ZN, Al-Zwairi AA. The relationship between tobacco smoking 406 and oral colonization with Candida species. J Contemp Dent Pract. 2010;11:017-24. 
407

408

409

410

411

412

27. NCCLS. Reference Method for Broth Dilution Antifungal Susceptibility Testing of Yeasts; Approved Standard-Second Edition. NCCLS document M27-A2 (ISBN 1$56238-469-4)$.

28. Barry AL, Brown SD. Fluconazole disk diffusion procedure for determining susceptibility of Candida species. J Clin Microbiol. 1996;34:2154-2157.

29. Urzúa B, Hermosilla G, Gamonal J, Morales-Bozo I, Canals M, Barahona S, Cóccola C, Cifuentes V. Yeast diversity in the oral microbiota of subjects with periodontitis: Candida albicans and Candida dubliniensis colonize the periodontal pockets. Med Mycol. 2008 Dec;46(8):783-93. doi: 10.1080/13693780802060899. PubMed PMID: 18608938.

30. Sheth CC, Makda K, Dilmahomed Z, González R, Luzi A, Jovani-Sancho Mdel M, Veses V. Alcohol and tobacco consumption affect the oral carriage of Candida albicans and mutans streptococci. Lett Appl Microbiol. 2016 Oct;63(4):254-9. doi: 10.1111/lam.12620. Epub 2016 Aug 25. PubMed PMID: 27450704.

31. Tanaka J, Tanaka M. Influence of type of prosthesis on oral environment and the number of missing teeth in elderly persons. Int J Dent. 2010; 22: PII: 584134.

32. Lie MA, Timmerman MF, Van der Velden U, Van der Weijden G. Evaluation of 2 methods to assess gingival bleeding in smokers and non-smokers in natural and experimental gingivitis. J Clin Periodontol 1998; 25:695-700.

33. Palmer RM, Scott DA, Meekin TN, Odell EW, Wilson RF. Potential mechanism of susceptibility to periodontitis in tobacco smokers. J Periodontal Res. 1999;34:363-369. 
428

429

430

431

432

433

434

435

436

437

438

439

440
34. Thein, Z. M., Samaranayake, Y. H. \& Samaranayake, L. P. Characteristics of dualspecies Candida biofilms on denture acrylic surfaces. J. Arch. Oral. Bio. 2007;52:12001208.

35. Bakki SR, Kantheti LC, Kuruba KK, Poosarla C, Baddam VR, Mulakaluri RR. Candidal carriage, isolation and species variation in patients undergoing radiotherapy and chemotherapy for head and neck tumors. J NTR Univ Health Sci 2014;3:28-34

36. Kleinegger CL, Lockhart SR, Vargas K, Soll DR. Frequency, intensity, species, and strains of oral Candida vary as a function of hostage. J Clin Microbiol 1996;34: 22462254.

37. Sardi JC, Duque C, Camargo GA, Hofling JF, Goncalves RB. Periodontal conditions and prevalence of putative periodontopathogens and Candida spp. in insulin-dependent type 2 diabetic and non-diabetic patients with chronic periodontitis - a pilot study. Arch. Oral Biol. 2011;56:1098-1105.

38. Barros L.M, Boriollo M.F, Alves A.C., Klein MI, Gonalves RB. Genetic diversity and exoenzyme activities of Candida albicans and Candida dubleniensis isolated from the oral cavity of Brazilian periodontal patients. Arch Oral Biol. 2008;53:1172-1178.

39. Jewtuchowicz VM, Mujica MT, Brusca MI, Sordelli N, Malzone MC, Pola SJ, Iovannitti CA, Rosa AC. Phenotypic and genotypic identification of Candida dubliniensis from subgingival sites in immunocompetent subjects in Argentina. Oral Microbiol Immunol. 2008 Dec;23(6):505-9. doi:10.1111/j.1399302X.2008.00465.x. PubMed PMID: 18954358. 
449

450

451

452

453

454

455

456

457

458

459

460

461

462

463

464

465

466

467

468

469

470

40. Javed F, Tenenbaum HC, Nogueira-Filho G, Nooh N, Taiyeb Ali TB, Samaranayake LP, Al-Hezaimi K. Oral Candida carriage and species prevalence amongst habitual gutka-chewers and non-chewers. Int Wound J. $2014 \mathrm{Feb}$;11(1):79-84. doi:

10.1111/j.1742-481X.2012.01070.x. Epub 2012 Aug 10. PubMed PMID: 22883719.

41. Reichart PA, Samaranayake LP, Samaranayake YH, Grote M, Pow E, Cheung B. High oral prevalence of Candida krusei in leprosy patients in Northern Thailand. J Clin Microbiol. 2002;40:4479-4485.

42. Järvensivu A, Hietanen J, Rautemaa R, Sorsa T, Richardson M. Candida yeasts in chronic periodontitis tissues and subgingival microbial biofilms in vivo. Oral Dis. 2004;10:106-112.

43. Williams DW, Walker R, Lewis MA, Allison RT, Potts AJ. Adherence of Candida albicans to oral epithelial cells differentiated by Papanicolaou staining. J Clin Pathol. $1999 ; 52: 529-531$.

44. De-La-Torre J, Quindós G, Marcos-Arias C, Marichalar-Mendia X, Gainza ML, Eraso E, Acha-Sagredo A, Aguirre-Urizar JM. Oral Candida colonization in patients with chronic periodontitis. Is there any relationship? Rev Iberoam Micol. 2018 Jul - Sep;35(3):134-139. doi: 10.1016/j.riam.2018.03.005. Epub 2018 Aug 3. PubMed PMID: 30082174.

45. Ye P, Wang X, Ge S, Chen W, Wang W, Han X. Long-term cigarette smoking suppresses NLRP3 inflammasome activation in oral mucosal epithelium and attenuates host defense against Candida albicans in a rat model. Biomed Pharmacother. 2019 May;113:108597. doi: 10.1016/j.biopha.2019.01.058. Epub 2019 
Mar 7. PubMed PMID: 30851547.

472

473

474

475

476

477

478

479

480

481

482

483

484

485

486
46. Chinnici J, Yerke L, Tsou C, Busarajan S, Mancuso R, Sadhak ND, Kim J, Maddi

A. Candida albicans cell wall integrity transcription factors regulate polymicrobial biofilm formation with Streptococcus gordonii. PeerJ. 2019 Oct 11;7:e7870. doi: 10.7717/peerj.7870. eCollection 2019. PubMed PMID: 31616604; PubMed Central PMCID: PMC6791342.

47. Tumbarello M, Tacconelli E, de Gaetano K, Ardito F, Pirronti T, Cauda R, Ortona L. Bacterial pneumonia in HIV-infected patients: analysis of risk factors and prognostic indicators. J Acquir Immune Defic Syndr Hum Retrovirol. 1998 May 1;18(1):39-45. PubMed PMID: 9593456.

48. Cartledge JD, Midgley J, Gazzard BG. Non-albicans oral candidosis in HIV-positive patients. J Antimicrob Chemother. 1999;43:419-422.

49. Rani M, Bonu S, Jha P, Nguyen SN. Tobacco use in India prevalence and predictors of smoking and chewing in a national cross-sectional household survey. Tob Control $2003 ; 12: 51-60$. 


\section{Figure 1}

Candida subspeciation in saliva samples from smokers with periodontitis Isolated saliva samples were centrifuged to pellet the microbial isolates which were then cultured on CHROMagar chromogenic media. The development of specific colored colonies helps in identifying the species of Candida within the sample.

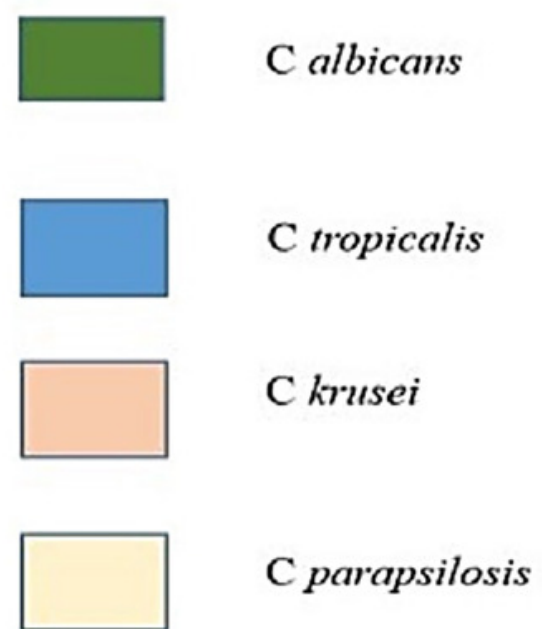

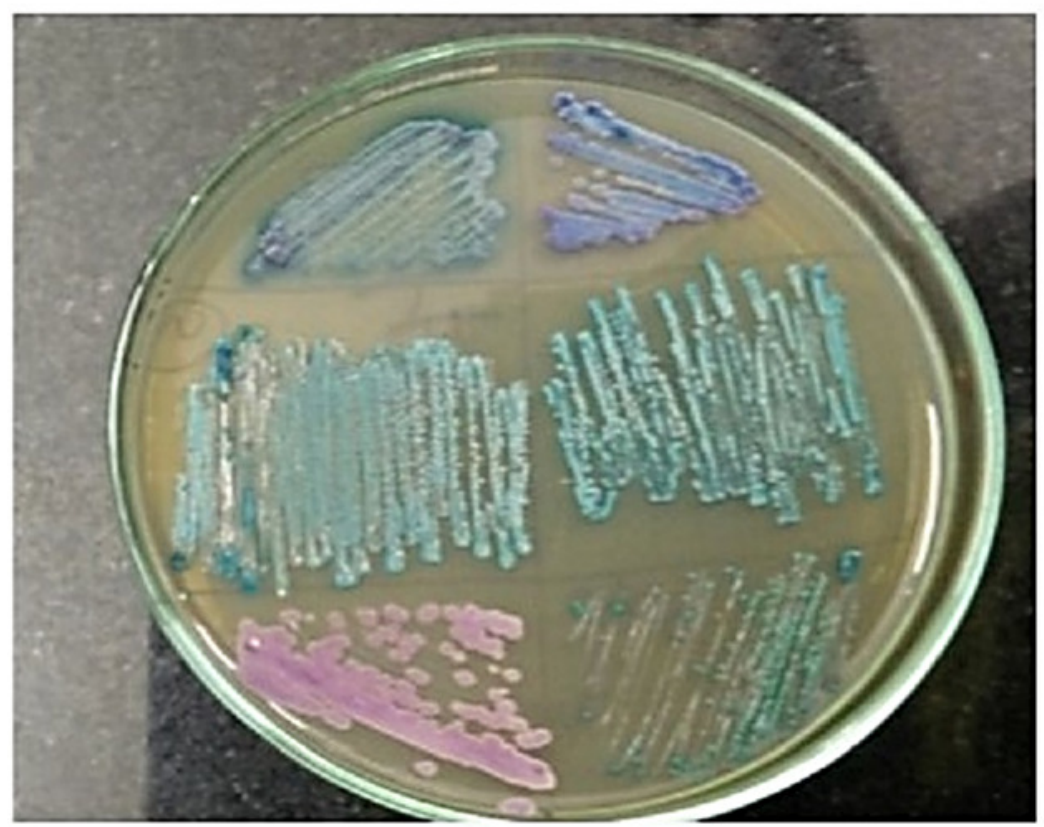


Figure 2

Correlation of Candida colonization of saliva with smoking and periodontitis.

Pearson correlation coefficient was calculated for understanding the correlation between the above characteristics.

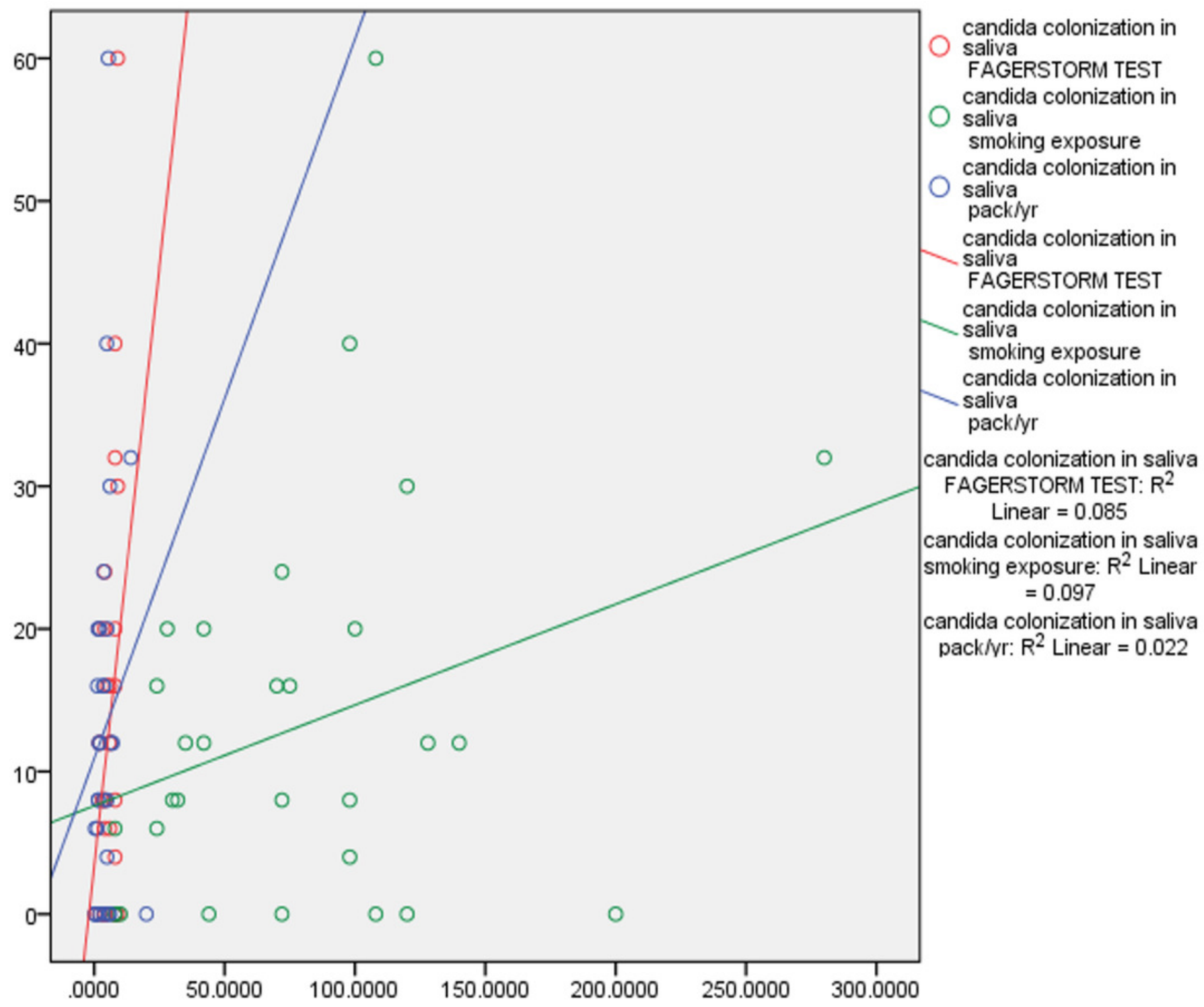


Figure 3

Correlation of Candida colonization of subgingival plaque with smoking and periodontitis.

Pearson correlation coefficient was calculated for understanding the correlation between the above characteristics.

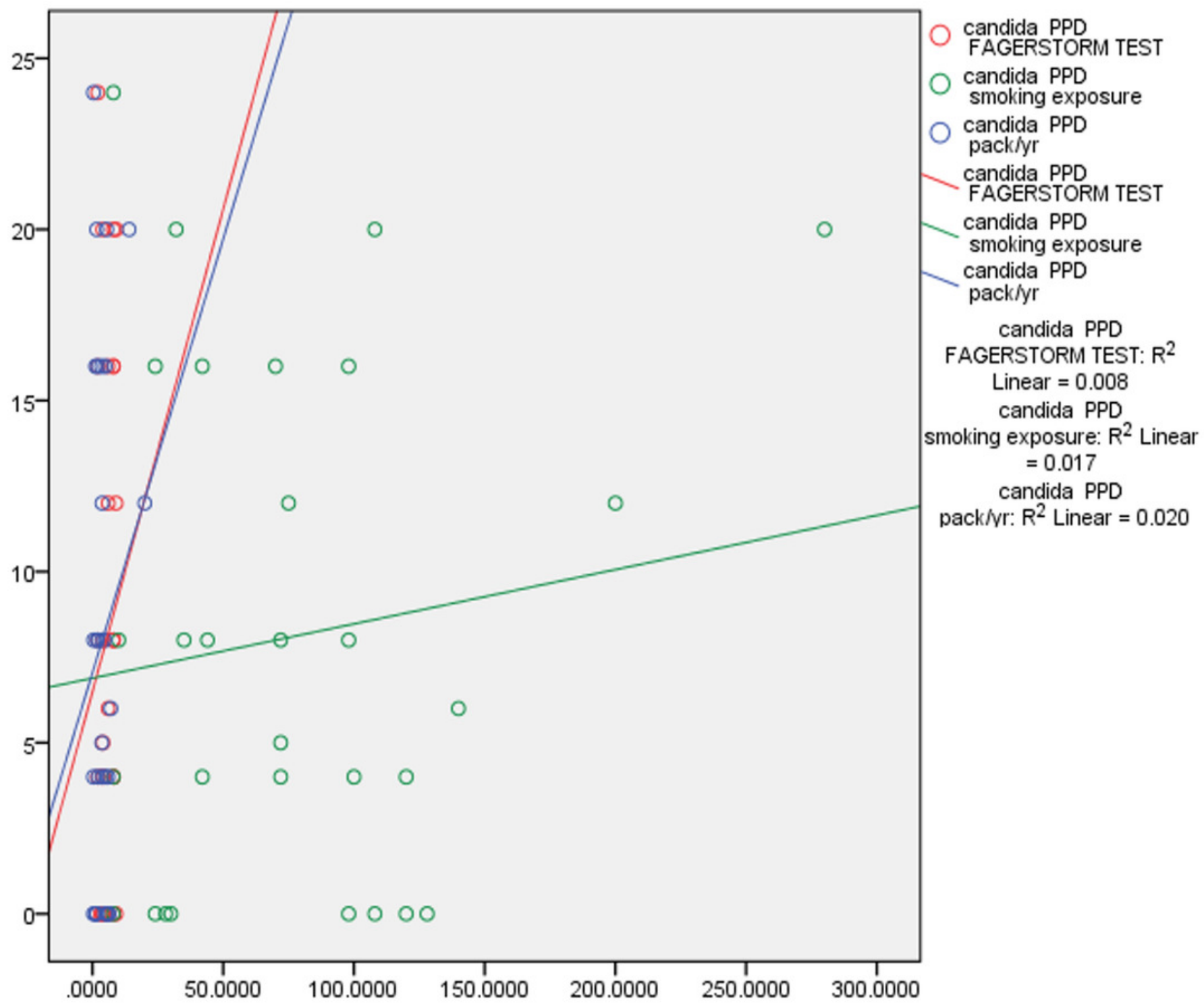




\section{Table $\mathbf{1}$ (on next page)}

Relative abundance of various Candida species within various groups for saliva and subgingival plaque samples.

Relative abundance of various Candida species within various groups for saliva and subgingival plaque samples Isolated samples were processed for determining $\mathrm{CFU} / \mathrm{ml}$ of various Candida species within groups 


\begin{tabular}{|l|l|l|l|l|l|l|}
\hline Candida Species & \multicolumn{2}{|l|}{ SALIVA } & \multicolumn{2}{l|}{ SUBGINGIVAL PLAQUE } \\
\hline & $\begin{array}{l}\text { Group 1 } \\
n(\%)\end{array}$ & $\begin{array}{l}\text { Group 2 } \\
n(\%)\end{array}$ & $\begin{array}{l}\text { Group 3 } \\
n(\%)\end{array}$ & $\begin{array}{l}\text { Group 1 } \\
n(\%)\end{array}$ & $\begin{array}{l}\text { Group 2 } \\
n(\%)\end{array}$ & $\begin{array}{l}\text { Group 3 } \\
n(\%)\end{array}$ \\
\hline C. albicans & $15(50)$ & $14(46.7)$ & $13(43.3)$ & $10(33.3)$ & $11(36.7)$ & $13(43.3)$ \\
\hline C. parapsilosis & $1(3.30)$ & 0 & 0 & 0 & 0 & 0 \\
\hline C. krusei & $3(10)$ & $4(13.30)$ & $3(10)$ & $7(23.30)$ & $4(13.30)$ & $3(10)$ \\
\hline C. tropicalis & $3(10)$ & $4(13.3)$ & $3(10)$ & $4(13.30)$ & $4(13.30)$ & $2(6.7)$ \\
\hline C. glabrata & $1(3.3)$ & 0 & 0 & $1(3.30)$ & $1(3.3)$ & 0 \\
\hline No Candida & $7(23.3)$ & $8(26.6)$ & $11(36.6)$ & $8(26.6)$ & $10(33.3)$ & $12(40)$ \\
\hline
\end{tabular}

1 n-no. samples positive for Candida species 


\section{Table 2 (on next page)}

Antifungal susceptibility of Candida isolates from subgingival plaque and saliva samples of various groups.

Isolated samples were processed for determining antifungal susceptibility for fluconazole and variconazole. F- Flucanazole treated; V-Voricanozole treated: $\mathrm{n}$ - number of samples; $\%$ - the percentage of samples that are either sensitive or resistant to antifungal agents. 
1

\begin{tabular}{|c|c|c|c|c|c|c|c|c|}
\hline \multirow[t]{3}{*}{ Candida Species } & \multicolumn{4}{|l|}{ SALIVA } & \multicolumn{4}{|c|}{ SUBGINGIVAL PLAQUE } \\
\hline & \multicolumn{2}{|c|}{ SENSITIVE } & \multicolumn{2}{|c|}{ RESISTANT } & \multicolumn{2}{|c|}{ SENSITIVE } & \multicolumn{2}{|c|}{ RESISTANT } \\
\hline & Count (n) & $\%$ & Count (n) & $\%$ & Count (n) & $\%$ & Count (n) & $\%$ \\
\hline C albicans (F) & 44 & 97.8 & 1 & 2.2 & 34 & 100 & 0 & 0 \\
\hline C albicans (V) & 44 & 100 & 0 & 0 & 34 & 100 & 0 & 0 \\
\hline C parapsilosis (F) & 1 & 100 & 0 & 0 & 0 & 0 & 0 & 0 \\
\hline C parapsilosis (V) & 1 & 100 & 0 & 0 & 0 & 0 & 0 & 0 \\
\hline C krusei (F) & 4 & 57 & 3 & 42.9 & 6 & 35.3 & 11 & 64.7 \\
\hline C krusei (V) & 6 & 100 & 0 & 0 & 17 & 100 & 0 & 0 \\
\hline C tropicalis (F) & 9 & 100 & 0 & 0 & 11 & 100 & 0 & 0 \\
\hline C tropicalis (V) & 9 & 100 & 0 & 0 & 11 & 100 & 0 & 0 \\
\hline C glabrata (F) & 0 & 0 & 0 & 0 & 2 & 100 & 0 & 0 \\
\hline C glabrata (V) & 0 & 0 & 0 & 0 & 2 & 100 & 0 & 0 \\
\hline
\end{tabular}

2 


\section{Table 3 (on next page)}

Karl Pearson's Correlation between Candida colonization in saliva and subgingival plaque and smoking.

Pearson's correlation coefficient ( $r$-value) was calculated for understanding the correlation between the above characteristics. Candida colonization was originally calculated based on $\mathrm{CFU} / \mathrm{ml}$ for each sample. 


\begin{tabular}{|l|l|l|l|l|}
\hline Parameters & \multicolumn{2}{|l|}{$\begin{array}{l}\text { Candida Colonization } \\
\text { in Saliva (r-value) }\end{array}$} & $\begin{array}{l}\text { Candida Colonization in } \\
\text { Subgingival Plaque (r-value) }\end{array}$ \\
\hline $\begin{array}{l}\text { Fagerstorm } \\
\text { Test }\end{array}$ & 0.291 (Fair) & 0.118 & 0.091 & 0.631 \\
\hline $\begin{array}{l}\text { Smoking } \\
\text { Exposure }\end{array}$ & 0.311 (Fair) & 0.094 & 0.311 (Fair) & 0.130 \\
\hline Pack Years & 0.150 & 0.429 & 0.141 & 0.141 \\
\hline PPD & -0.100 & 0.447 & -0.022 & 0.868 \\
\hline CAL & -0.57 & 0.663 & 0.038 & 0.773 \\
\hline PPD - Probing Pocket Depth; CAL - Clinical Attachment Loss. \\
\hline
\end{tabular}

1 